\title{
750KW Wind Turbine Generator System Gearbox Dynamics Simulation
}

\author{
Analysis \\ Wei Jia ${ }^{1, a}$, Yang Zhang ${ }^{1, b}$, Hui Dou ${ }^{1, \mathrm{c}}$, and Hua Yan ${ }^{1, \mathrm{~d}}$ \\ ${ }^{1}$ XJELECTRICCO., LTD, Xuchang, 461000, China \\ azzzx_jiawei@126.com, bzzzx_zhangyang@126.com, czzzx_douhui@126.com, \\ dzzzx_yanhua@126.com
}

KeyWords: wind turbine ; gearbox; gear; dynamics simulation analysis

Abstract. In the operation of Wind turbine generator system has the fierce system vibration, which has the very tremendous influence on gearbox's movement precision and gear's life. As for the complexity of gearbox's structure and working conditions, it is necessary to research on the vibrate question of wind turbine gearbox. To this situation of gearbox , this paper designed and modeled again. This paper based on multibody system dynamic method, modality vibration, impact-contact theory, taked $750 \mathrm{KW}$ wind turbine gearbox as the research object. Through the simulation analysis of gearbox, obtains the gear the impact force as well as the kinetic energy along with the time variation curve. This paper become the high speed gear shaft the flexible body to carry on the stress analysis, obtains the high speed gear shaft's stress distribution map for the gearbox optimization design in the future has laid the foundation.

\section{Introduction}

With the continuous economic development, the environmental pollution question is valued people's more and more. The wind energy takes one kind of clean energy even more to take seriously by the people. The development wind power generation already became our country significant developmental strategy. The wind turbine is the humanity uses the wind energy the major installation at present. The gearbox has been taken as wind-driven generator's key component by the people. Because the wind energy is the uncontrolled system energy, the air blower in the movement period wind direction and the wind speed is change timely, this causes very difficultly to describe gearbox's stress situation precisely. The wind turbine is rigid and flexible coupling multibody systems, the wind turbine work in bad environment, the function in wind turbine loads and so in air blower's aerodynamic force, force of inertia and elastic force will cause the system to have the distortion or the vibration, Affects wind turbine's normal operation, Cause serious damage to gearbox's, which led to the high failure rate gearbox. In order to guarantee that wind turbine's stability and the reliability, make a deep research on gearbox has a great practical significance.

\section{The principle of multi-rigid body multi-flexible body systems dynamics analysis}

The establishment of Rigid dynamics equation. Lagrange multiplier establishment equation:

$$
\frac{d}{d t}\left(\frac{\partial T}{\partial \phi}\right)^{T}+f_{q}^{T} \rho+g_{\& \alpha}^{T} \mu=Q
$$

Complete restraint: $f(q, t)=0$ Incomplete constraint: $f(q, \& t)=0 . T$ is unit system's function and $q$ is system generalized coordinates array $q=\left[q_{1}, q_{2}, q_{3}, \ldots, q_{n}\right]^{T}, q$ is system generalized velocity array, $Q$ 
is generalized force array; $p$ is holonomic bond Lashi multiplier array, $\mu$ is non-holonomic bond Lashi multiplier array.

Multi-flexible body systems dynamics equation. The flexible body random point's position vector expression:

$$
r_{p}=r_{B}+A\left(s_{p}+u_{p}\right)
$$

$r_{B}$ is local reference frame for the flexible body coordinate system origin location in the overall loss of volume, the component is $\mathrm{x}, \mathrm{y}, \mathrm{z} ; s_{p}$ is the point has not distorted when in partial reference system's radius vector, the component is a constant, $\mathrm{A}$ is the partial reference coordinate system to the overall coordinate system's transformation matrix, the position uses the Euler angle $\psi, \theta, \phi$ expression; $u_{p}$ is distorts after the point position before distortion position vector.

the flexible body's equation of motion with the Lagrange multiplier:

$\left\{\begin{array}{l}\frac{d}{d t}\left(\frac{\partial L}{\partial \xi^{\&}}\right)-\frac{\partial L}{\partial \xi}+\frac{\partial \Theta}{\partial \xi^{\Phi}}+\left[\frac{\partial \psi}{\partial \xi}\right]^{T} \lambda-Q=0 \\ \psi=0\end{array}\right.$

L is lagrange function; $\Theta$ is the energy consumes energy; $\Psi$ is Constraint equation; $\lambda$ is lagrange multiplier.

\section{Gearbox design}

Gearbox transmission type choice. At present, the $750 \mathrm{kw}$ wind turbine generator system gearbox structural design mainly has two forms: one is one level planet, one level parallel axis structure design, Namely RENK structure , another is FLEND structure, one level planet and two level of parallel axis structures. Because the design requirements whole transmission ratio reach 67.4, Consider the overall structure of the gearbox, this design choose one level planet and two level parallel axis structure pattern,the whole transmission ratio is 67.4. The planet structure use the heart piece fluctuation, supposes various gears material, tooth flank degree of hardness to be the same. Take the planetary gear transmission ratio is 5.04, the dead axle transmission partial velocity ratio is 13.4. The wind rotor through the main axle input gearbox, after undergoing the acceleration, transmits the generator. Its diagram of mechanism as shown in Fig.1.

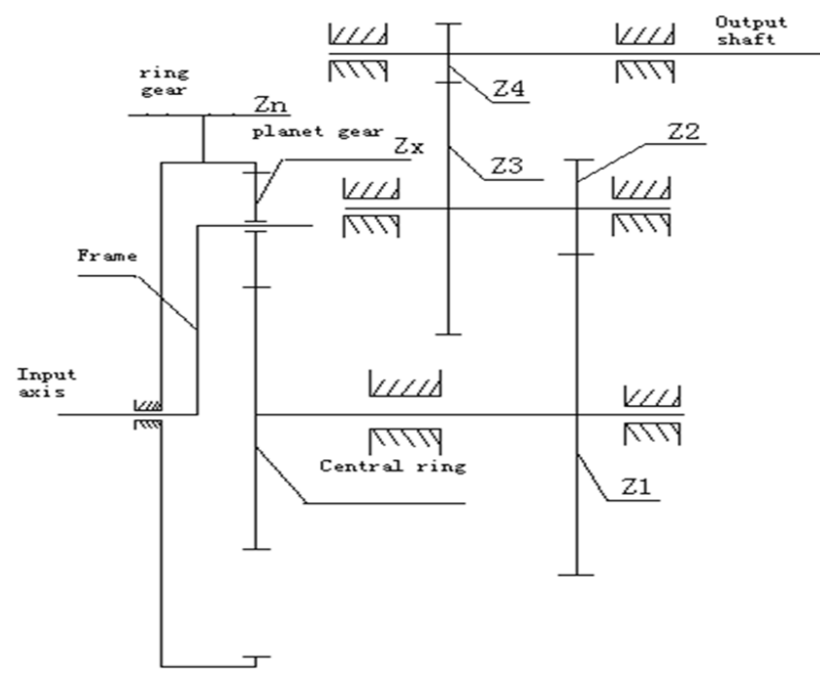

Fig.1 gear diagram of gearbox

750KW gearbox model establishment. In gearbox's preliminary design, needs to determine the basic structure and the main parameter, and check the strength of the main components in order to ensure the stability of the gearbox. The main axle uses the double bearing arrangement to guarantee 
that axial and the radial load caused by the wind rotor does not transmit in the gearbox. In order to reduce the vibration, the gearbox use torque arm installs in the engine room in the elastic support. The input axis through contracts the plate and the main axle joint, the output shaft passes the flexible coupling and the electrical machinery is connected. According to determined that the good parameter counter gearbox carries on the model shown in Fig. 2 and Fig. 3.

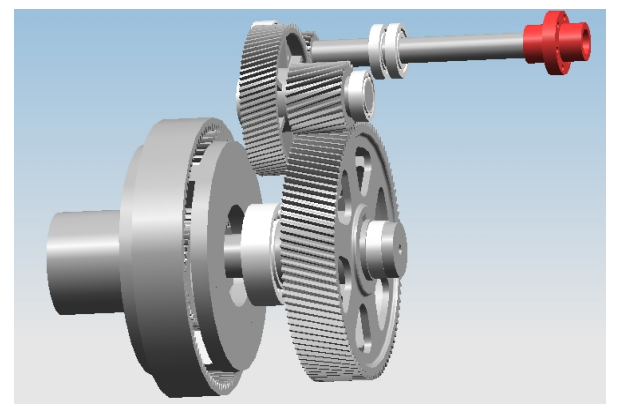

Fig. 2 The main transmission gearbox parts

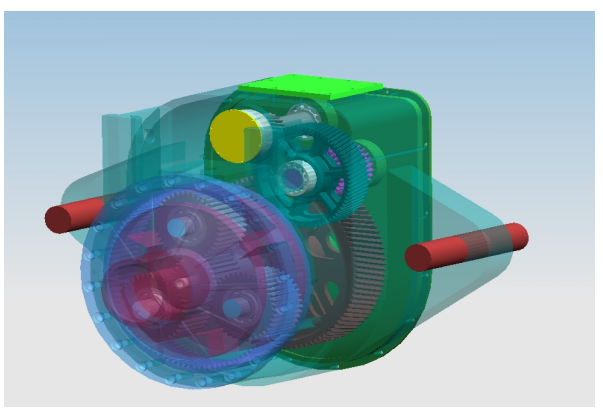

Fig. 3 Gearbox Model

\section{Gearbox's dynamics simulation analysis}

On the planet rack, there exerts a permanent rotational speed in the establishment rigid body model's input axis. The size is 135 degree/s namely $22.5 \mathrm{r} / \mathrm{min}$ simulates the wind wheel to transmit the speed-up box's rotational speed, exerts constant load moment $4976053 \mathrm{~N}$.mm on the output shaft to simulate the generator to system's torque. Obtains the simulation result in ADAMS.

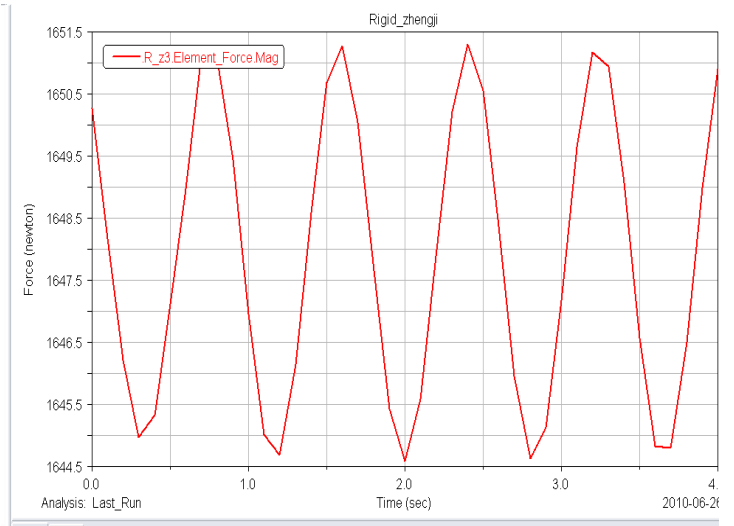

Fig. 4 gear 3 impact forces

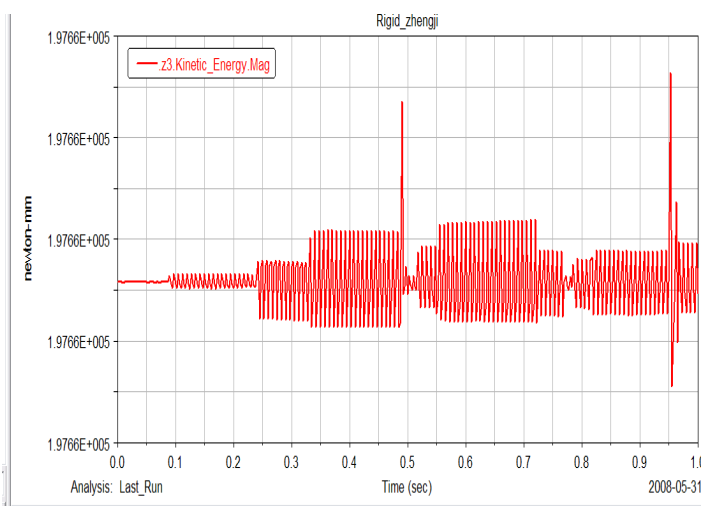

Fig. 6 gear 3 kinetic energy curves

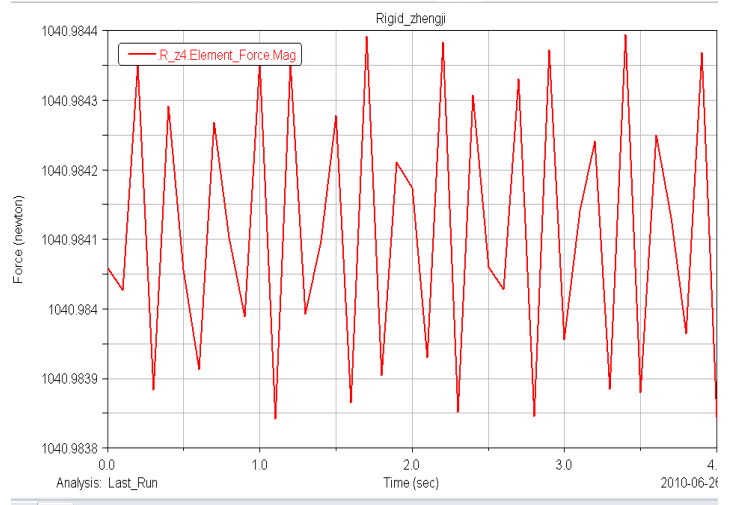

Fig. 5 gear 4 impact forces

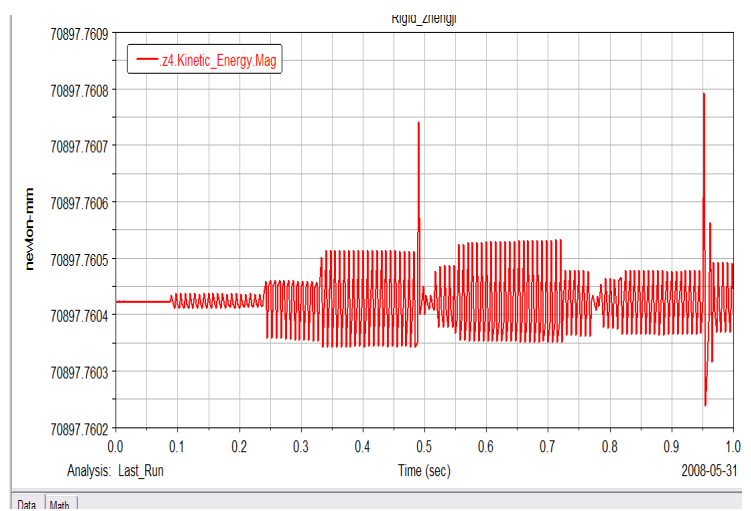

Fig.7 gear 4 kinetic energy curves

From Fig. 4 and Fig. 5 can discover that Z3 and Z4 impact force simulation computation's gear exciting force about fluctuates is big, sometimes probably reach the average value 2-3 times. It is consistent with the conclusion that gear drive coincidence degree due to mesh stiffness changes caused large changes in excitation. In the gear meshing force include dynamic load components, and the meshing force around a fixed value fluctuations.It indicated that the process of gear in the 
transmission obvious shock and vibration. In addition, when the driven gear 1 speed increases, the transmission process suffered shock and vibration is increasing. From the gear kinetic energy curve we can be found that the gear kinetic energy change around a constant fluctuation, and there is a certain periodicity. The simulation results above reflect accurately the environment in the constant impact force and the kinetic energy of all gear changes.

\section{The high speed gear shaft flexible body analysis}

The gearbox model we established, its component belongs to the rigid body, in MSC.ADAMS for motion analysis in the elastic deformation will not occur. However, the gearbox will be will occur distorts and moves the change, will also have the intense vibration production in actual movement.so making the mechanical analysis must consider the flexible effect. Especially high speed gear shaft $\mathrm{z} 4$ its rotational speed reaches as high as $1520 \mathrm{r} / \mathrm{min}$. Therefore, this paper will become the high speed gear shaft production to flexible body. The gearbox is carried a rigid and flexible coupling body on the research. The MSC.ADAMS flexible module is uses the modality to express the object elasticity, Its main idea entrusts with a flexible body modality collection, expresses the elastic displacement with the modality vector and the modality coordinate's linear combination, through calculates each time object the elastic displacement to describe its amoeboid movement. Carries on the simulation according to the actual operating mode counter gear transmission, obtains stress cloud chart which shown in Fig. 8.

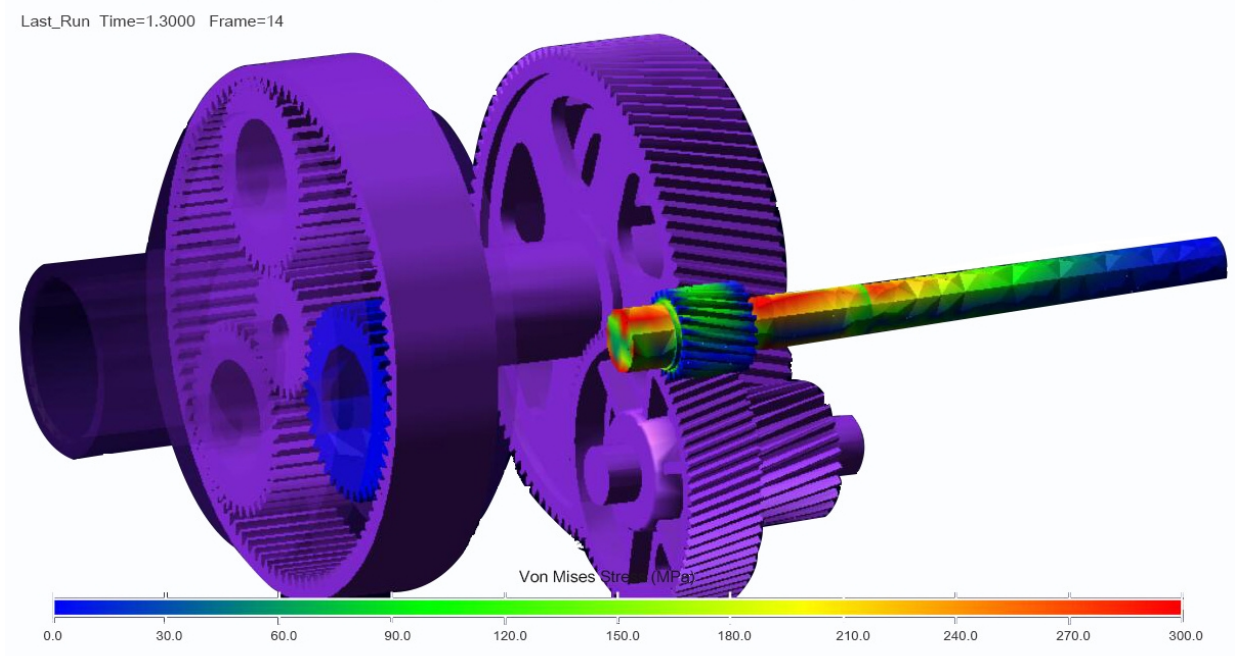

Fig. 8 stress cloud chart

Fig. 8 shows that high speed axis $\mathrm{z} 4$ and the $\mathrm{z} 3$ contact's nearby stress is quite big ,the biggest stress has reached $355 \mathrm{MPa}$, is smaller than the high speed axis allowable stress. Obviously, we also find the stress which from the chart the high speed gear shaft receives is bigger many than the planetary gear. It is consistent with the actual that the high-speed gear shaft damage the most serious during the operation. When we doing optimization of gearbox design, we should focus on consideration it in future.

\section{Conclusion}

This paper view of common failure form of domestic 750kw gearbox, the parameters of the gearbox has been redesigned, and carries on the model of gearbox through the UG. Carries on dynamics simulation using the MSC.ADAMS software counter gearbox to obtain the gear meshing impact force and the kinetic energy curve change chart, has discussed gearbox's high breakdown reason.To high fault high speed gear shaft, this paper become the high speed gear shaft the flexible body to carry on the stress analysis, obtained high speed gear shaft's stress distribution map. By analyzing 
the stress distribution to high-speed gear shaft to explain the reasons for failure foundation for dynamics of the whole gear box and gearbox optimal design in future.

\section{References}

[1] Wang Jielong, Chen Yan, Xue Kezong. Large-scale horizontal axis wind turbine stability[J]. Engineering Mechanics, 2002,19 (2) :83-86.

[2] HeJing, HeYuLin, JinXin, LiuHua. Stall wind turbine system vibration and simulation analysis[J]. Chongqing University Technology, 2007,30 (7) :91-95.

[3] Guoyu Zhang:Numerical Simulation of Aaerodynamic Performance for Wind Turbines. East China Electric Power(2009) 